\title{
Osteoporosis and arthritis
}

If hypertension is a silent killer, osteoporosis is a silent thief. It insidiously robs the skeleton of its banked resources, often for decades, until the bone ultimately becomes weak enough to sustain a spontaneous fracture. ${ }^{1}$

Recently, the field of metabolic bone disease has received prominent recognition because of the increased public awareness of the prevalence and problems associated with osteoporosis. Because of the enormous disability and health care costs resulting from this disease, attempts have been made to try to identify subjects at risk or those who have established disease for either preventive or therapeutic interventions.

Whatever orientation the rheumatologist has chosen as a career-pure immunology, inflammatory diseases in general, or aches and pains syndromes of the musculoskeletal system-there will be confrontation with osteoporosis, either because of the side effects of treatment (corticosteroids), or because of secondary effects of the disease on bone (rheumatoid arthritis (RA)), or because of primary bone rarefaction (vertebral crush fracture).

\section{Definition of osteoporosis}

The classical definition of osteoporosis by Albright and Reifenstein ${ }^{2}$ - 'too little bone, but what bone there is, is normal'-has been widely accepted. This fundamental definition has never seriously been challenged up to now, but this may change in the near future. This definition, however, made a clear distinction between osteoporosis and osteomalacia. In osteomalacia the ratio of mineral to organic matrix is low, indicating a mineralisation defect, which is generally due to a fault in vitamin D metabolism. Albright's definition does not, however, define 'too little bone'. If this means too little by the standards for adults in the prime of life, many people without symptoms have osteoporosis simply because the physiological changes of aging have affected their bones.

Osteoporosis is defined clinically as loss of bone (osteopenia) to an extent sufficient to result in fracture with minimal trauma, generally of the spine, but also of the hip and wrist. Long before the fractures are manifest radiologically the patient has unknowingly already progressively lost $30-50 \%$ of his or her bone capital over the previous 10-20 years.

Although osteoporosis is the most common of the diseases that affect bone, the condition, as a well defined disease entity, remains problematic. The reason for this is that differentiation between physiological bone loss as part of the aging process (osteopenia) and pathological bone loss (osteoporosis) is often difficult. In practice, this leads either to overdiagnosis, where everyone above the age of 50-60 is thought to have osteoporosis and backache after the menopause is also attributed to this cause, or to late diagnosis, where osteoporosis is recognised only when a spine, hip, or wrist fracture occurs.

Osteoporosis at present is not considered to be a disease but a bone failure syndrome, disclosed by a fracture from minimal trauma in a person with reduced skeletal mass. Analogous syndromes include congestive heart failure, renal insufficiency, hepatic failure, mental deficiency, arterial insufficiency, and respiratory insufficiency. All these syn- dromes have a commmon physiopathology-namely, insufficient organ reserves to meet intercurrent minor stress.

Because of the confusion in the use of the term osteoporosis the National Institute of Health (USA) Consensus Conference on Osteoporosis in 1984 formulated the following definition for osteoporosis. Primary osteoporosis is an age related disorder characterised by decreased bone mass and by increased susceptibility to fracture in the absence of other recognisable causes of bone loss. Secondary osteoporosis is then the condition in which osteoporosis and fractures are the result of excessive bone resorption in specific endocrinopathies and arthropathies, or of excessive and prolonged use of drugs which interfere with bone metabolism, as for example corticosteroids.

\section{Identification of those who are at risk}

If osteoporosis is to be prevented the first step is to identify those at risk in good time. The bone depleting effect of oestrogen deficiency is well reported. Patients at high risk for primary and secondary osteoporosis are women with premature oestrogen deficiency, whether of surgical or natural origin, who clearly require oestrogen substitution treatment. Knowledge of factors other than oestrogen deficiency that are likely to cause or aggravate bone loss related to age and sex is of clinical value for prediction and counselling purposes.

\section{CLINICAL CRITERIA}

There seems to be general agreement that small, thin, fair, pale skinned women (especially those of northern European extraction), spinsters or mothers of only one or two children, those with strong family histories of hip and spine fracture, and those who, in addition to the foregoing criteria, are heavy smokers run an appreciably higher fracture risk (table).

It is of clinical interest to note that osteoporosis and generalised osteoarthrosis are rarely seen together, though both are common in the older age group. Current data suggest that there are real differences between the two conditions. ${ }^{3}$

As the first clinical signs of generalised osteoarthrosis (Heberden and Bouchard nodes) are readily seen in the hand joints in the perimenopausal period, these can be useful in eliminating a group of women who are probably not at risk of developing symptomatic osteoporosis and thus do not need preventive treatment.

\section{OBJECTIVE CRITERIA}

Objective identification of the group at risk of fracture is a goal of great practical significance as treatment of an unnecessarily large group would be difficult, expensive, and, in certain instances, fraught with needless risk.

Refinements in bone mass measurement techniques readily available in medical practice, such as radiogrammetry, single and dual photon absorptiometry, as well as dual energy absorptiometry, would help to identify preosteoporotic women with a low bone mass.

In the 1960s radiogrammetry of the second metacarpal, and in the 1970s single photon absorptiometry of the radius, 
Risk factors for osteoporosis

Genetic:
-Women more than men
- Whites more than blacks
-Family history of osteoporosis fracture
-Absence of generalised osteoarthritis
Anthropometric:
-Small stature
-Fair, thin, pale-skinned
-Slenderness
Hormonal:
-Premature menopause: natural, iatrogenic
- Nullipara
Dietary:
-Low diet: adolescence, menopause
-Excess proteins ${ }^{2}$
Lifestyle:
-Sedentary life
-Alcohol abuse
-Smoking
Concurrent illness and drugs:
-Gastrectomy, hyperparathyroidism
- Hyperthyroidism, rheumatoid arthritis
- Corticosteroids
- Neurological disease: cerebrovascular accident, Parkinson's
disease, etc

were developed, allowing reasonably accurate measurement of cortical bone mass in the forearm. These methods have proved very useful for epidemiological research and group follow up measurements. ${ }^{4}$

Some fractures due to postmenopausal osteoporosis affect areas of predominantly trabecular bone in the spine, however, and there is now abundant evidence that forearm bone mass measurements are poor predictors of vertebral crush fractures, but more reliable for femoral neck fractures, which occur in a predominantly cortical bone area. ${ }^{5}$

Two new fundamentally different techniques_namely, dual photon absorptiometry and dual energy absorptiometry, on the one hand, and computed tomography, on the other, now enable measurement of bone mass in areas affected by postmenopausal osteoporosis. These newer methods allow corrections for soft tissue absorption and thus permit bone density measurement in such areas as the lumbar spine and proximal femur. Sensitive and accurate methods of assaying bone mass in selected skeletal regions that yield clinically useful results are now widely available. The main purpose of such measurements will be to detect people already at high risk for fracture.

If measurements in the radius are used for screening it should be realised that a low radial bone mineral content indicates a high probability of concomitant low skeletal calcium and low spinal bone mineral content, though a normal value does not exclude spinal osteoporosis. Radial bone mineral content may be of more value in predicting bone loss in the hip than in the spine, whereas bone mineral measurement of trabecular bone in the spine will give a better indication of those who are at risk for vertebral crush fractures.

There is an accumulating body of evidence to indicate that there is a threshold value for vertebral fractures. This corresponds to a bone mineral content about two standard deviations below the mean adult peak bone mass (fig 1 ).

Many studies express results as the bone mass per unit area or volume (apparent density) to enable comparison, but the strength of the bone depends on its total mass (and internal architecture) rather than its density. There are minor sex differences in the apparent density of trabecular bone segments. The sex differences in fracture patterns, as in the case of strength, must reflect differences in bone size.

In our experience bone mass at the lumbar spine in patients with osteoporosis with vertebral crush fractures was comparable in men and women. This provides strong

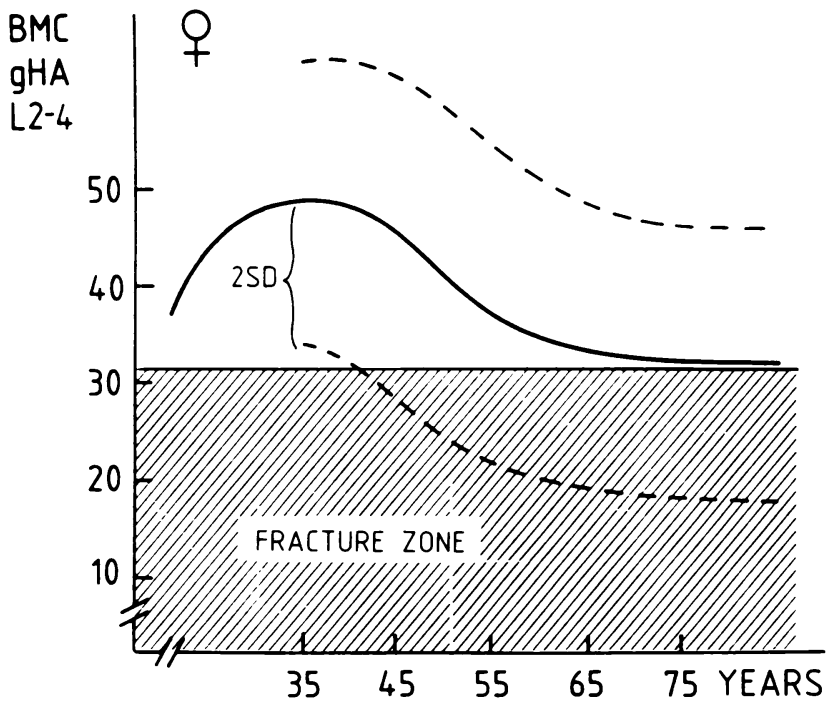

Figure 1: Lumbar spine fracture threshold in relation to age. $B M C=$ bone mineral content, $g H A=$ grams hydroxyapatite.

evidence for the validity of a fracture threshold at the lumbar spine. Female patients with vertebral crush fractures had a greater reduction of lumbar spine bone mineral content than radial bone mineral content, whereas the reverse was true for patients with femoral neck fractures. Furthermore, total bone mineral content at the lumbar spine (grams hydroxyapatite) was better than the area density (grams hydroxyapatite $/ \mathrm{cm}^{2}$ ) in separating women with spinal fractures from healthy controls matched for age and sex. ${ }^{6}$

\section{Bone metabolism in rheumatoid arthritis}

Bone loss has been recognised as a complication of the rheumatoid process for more than a century. Barwel was the first to apply the term 'osteoporosis' to the bone disease in RA. ${ }^{7}$ This osteoporosis, as recognised by Soila, ${ }^{8}$ McConkey et al, ${ }^{9}$ Saville and Kharmosh, ${ }^{10}$ and Kennedy and Lindsay, ${ }^{11}$ may be localised, occurring close to the site of inflamed joints, or generalised, involving the whole skeleton.

There have been a number of controversial reports about the systemic effect of RA on bone metabolism. Parathyroid overactivity and hypercalcaemia, as well as osteomalacia, have been described as part of the rheumatoid process. Rheumatoid arthritis affects more women (premenopausal and postmenopausal) than men and leads to chronic immobility in some but not in others. It is accompanied by serum protein alterations and requires a variety of drug treatment regimens. All these factors have an influence on bone remodelling indices, so that evaluation of the effect of RA is complicated and very difficult. ${ }^{12}$ For these reasons we studied prospectively bone metabolism indices in a homogeneous group of postmenopausal women who were in hospital owing to an exacerbation of their disease.

Patients with RA who had never had steroid treatment differed from the control population as they had significantly higher serum phosphorus concentrations, alkaline phosphatase activity, and osteocalcin concentrations. Calcitropic hormone concentrations (parathyroid hormone, 25-hydroxyand 1,25-dihydroxyvitamin D concentrations) were normal. Serum calcium concentrations were also normal when corrected for serum albumin. Excretion of urinary hydroxyproline and glycosaminoglycan was significantly increased. ${ }^{13-15}$ These biochemical data indicate that in postmenopausal patients with $\mathrm{RA}$ there is an increase in metabolic bone activity. This observation is in agreement 
with whole body technetium $\left({ }^{99 \mathrm{~m}} \mathrm{Tc}\right)$ retention studies, which showed a generalised increase in bone turnover in patients with RA not treated with steroids. ${ }^{16}$

Using single and dual photon absorptiometry in postmenopausal patients with RA, we found a significantly reduced peripheral bone mineral content at the radius, but a normal bone mineral content in the lumbar spine. ${ }^{17}$ Similarly, using dual quantitative computed tomography, we found no decrease in pure trabecular bone density in postmenopausal women (Dequeker and Van Holsbeek, unpublished data). In a recent study, however, low trabecular bone density was found in a small number of young patients with RA. ${ }^{18}$ In young patients with RA the same group also found low trabecular bone volume at the iliac crest. ${ }^{19}$ Although in RA the data suggest a higher metabolic bone activity, no increased bone loss in the vertebral column was detected, possibly owing to inhibition of prostaglandin $E_{2}$ synthesis by non-steroidal anti-inflammatory drugs. Massive osteolysis in RA occurs rarely and is usually associated with other concomitant diseases. ${ }^{20}$

Corticosteroids and the skeleton in rheumatoid arthritis The question of whether or not, or to what extent, corticosteroids produce or enhance the osteoporosis of RA has been much debated. ${ }^{21}$ There is no doubt that high doses of corticosteroids, $>7.5 \mathrm{mg}$ prednisone daily, are deleterious for the skeleton at all ages in a number of cases but more so in children and after the menopause. ${ }^{22-24}$

Low dose corticosteroid treatment in our hands did not seem to alter the bone mineral content in the peripheral or in the axial skeleton. ${ }^{17}$ The latter finding is surprising as in the group treated with corticosteroids a significantly $(p<0.01)$ higher incidence of fractures of the vertebrae or femoral neck occurred.

Other authors, using subjective density grading of $x$ rays of the spine, have also been unable to show significant differences between patients with RA treated or not treated with corticosteroids despite the occurrence of more vertebral fractures in the treated group. ${ }^{25}$

The explanation for these contradictory observations-no corticosteroid induced changes in bone mineral content in the spine but a high incidence of fractures in the group treated with corticosteroids-is not clear, but possibly, corticosteroids alter the quality of the bone rather than the quantity of bone mineral.

The mechanism by which corticosteroids may alter bone quality is unknown. Low doses of prednisone (5 mg daily) do not affect vitamin D metabolism, ${ }^{26}$ but high doses may lead to secondary alterations in 25-hydroxyvitamin D metabolism ${ }^{27}$ and to a marked decrease in intestinal calcium absorption even in the presence of normal 25-hydroxy- and 1,25-dihydroxyvitamin D concentrations.

Another factor which may play a part in the pathogenesis of steroid induced fractures is the well known antianabolic effect of corticosteroids, which is particularly evident in children, in whom skeletal growth may decrease. In vitro studies have shown that glucocorticoids directly inhibit bone formation and thus reduce the synthesis of all major matrix components, including collagen and proteoglycans. Because of the feedback inhibition of adrenocorticotrophic hormone secretion, androgen production in the surrenals and ovaries is reduced and thus the main source of oestrogens after the menopause by aromatisation of androstenedione to oestrone is eliminated. ${ }^{28}$

\section{Prevention and treatment of corticosteroid induced bone disease}

Treatment or prevention of corticosteroid induced bone disease is directed towards improving intestinal calcium absorption by giving vitamin D. (50 000 units once or twice weekly) and supplemental calcium (1.0-1.5 g daily, preferably at night). Studies have shown that near-physiological doses of 1,25-dihydroxycholecalciferol $(0.4 \mathrm{mg}$ daily) can increase calcium absorption in patients receiving high doses of glucocorticoids. ${ }^{29}$ Monitoring of serum and urinary calcium concentrations is imperative. If urinary calcium exceeds $300 \mathrm{mg} /$ day the dietary calcium intake should be reduced.

The above approach will at least partially correct the high rate of bone resorption secondary to the decline in intestinal calcium absorption, but it will not correct the depressed rate of bone formation. ${ }^{24}$ Sodium fluoride $(50-100 \mathrm{mg}$ daily) is known to stimulate trabecular bone formation, and, according to Baylink, this stimulation occurs even when pharmacological doses of prednisone are being given. ${ }^{30} \mathrm{~A}$ rational therapeutic approach in patients with glucocorticoid induced bone disease is thus a combination of sodium fluoride, vitamin D, and calcium. This combined treatment, however, does not increase bone mass in the appendicular skeleton, and a decrease in cortical bone has been found which may result in cortical bone fracture at the femoral neck. About $33 \%$ of fluoride treated patients develop adverse reactions (nausea, arthralgia, and fasciitis).

Although combined treatment with sodium fluoride, vitamin $\mathrm{D}$, and calcium is promising, it requires long term evaluation and monitoring of peripheral and axial bone mass.

In addition, if bone disease is severe, correction of any oestrogen or androgen deficiency in women should be considered. As anabolic steroids with low androgenic activity have proved effective in increasing peripheral bone mass and arresting accelerated bone loss in idiopathic osteoporosis $^{31} 32$ they are worth a therapeutic trial in cases where other agents, especially sodium fluoride or oestrogen, are ineffective or not tolerated.

\section{Mediators of bone destruction and repair in chronic arthritis}

Pathogenic mechanisms in bone destruction and repair in arthritis involve interactions between cells intrinsic to bone and infiltrating inflammatory cells. The actions of inflammatory cells on bone may include: $(a)$ modification of the release of mediators derived from bone cells; $(b)$ alteration of bone cell responsiveness to local and systemic hormones; (c) production of non-physiological mediators acting on bone cells; and (d) direct actions on bone matrix.

Although the local regulatory mechanisms of normal bone remodelling are not fully understood, much information on bone cell interaction has been derived from studies of bone cells in vitro. There are many stimulators and inhibitors of osteoclastic function, some of which are shown in fig $2 .{ }^{33}$

The mechanism of bone turnover is complicated, entailing communication between several cell types, which respond to systemic hormones and locally released factors. The sequence of successive activation of bone cells is started by recruitment of bone resorbing osteoclasts to the remodelling site. The resorption phase is followed by the arrival of osteoblasts, which replace the bone excavated by the osteoclasts. Mononuclear cells of the monocyte/macrophage lineage may be involved in the coordination of the two phases. The rates of bone resorption and formation are closely related, and this tight 'coupling' is conserved under many pathological conditions. Coupling of the catabolic and anabolic phases of bone remodelling may also explain the ability of the major bone resorbing hormones, parathyroid hormone and 1,25-dihydroxyvitamin $D_{3}$, to stimulate bone formation. Clearly, the concept of bone cell coupling is 
STIMULATORS

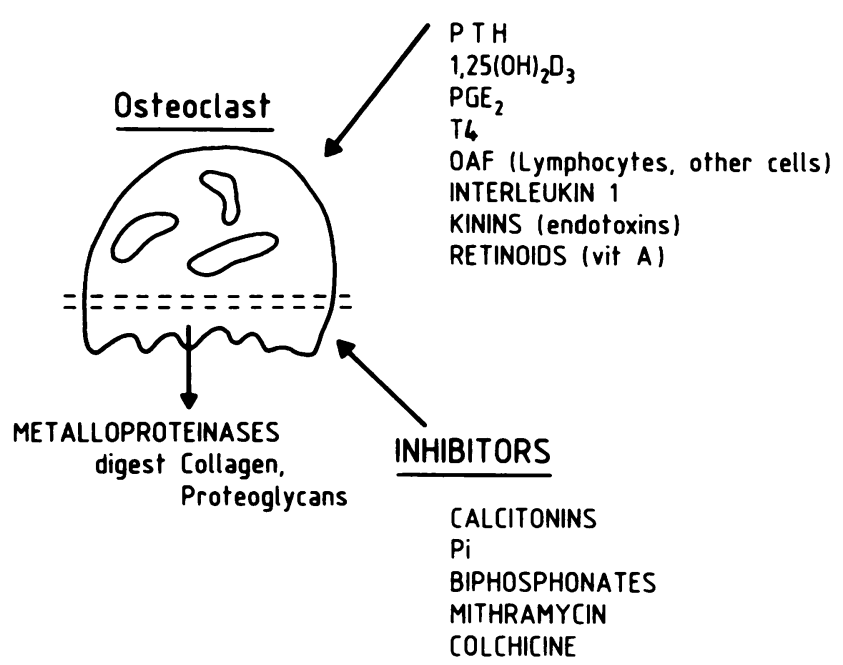

Figure 2: Activators and inhibitors of osteoclasts function assessed by calcium mobilisation and matrix degradation. $P$ TH=parathyroid hormone; $1,25(\mathrm{OH})_{2} D_{3}=1,25$-dihydroxyvitamin $D_{3} ; P G E_{2}=$ prostaglandin $E_{2}$, $T 4=$ thyroxine; $O A F=$ osteoblast activating factor; $P i=$ inorganic phosphate.

important for the interpretation of inflammatory tissue reactions in bone.

In addition to direct cell contact, interactions of the osteoblast and osteoclast lineages may entail the release of messenger molecules. Bone surface lining cells may generate signals governing the recruitment of osteoclasts. This hypothesis is strengthened by the observation that osteoblasts, but not osteoclasts, express receptors for the bone resorbing hormones, parathyroid hormone and 1,25dihydroxyvitamin $\mathrm{D}_{3}$. Moreover, matrix constituents are potent chemoattractants for bone resorbing cells, and osteoblasts may 'uncover' the resorption site by hormone regulated release of matrix degrading enzymes. Similarly, the recruitment and proliferation of osteoblasts following resorption may depend on autocrine or paracrine growth factors positioned in the bone matrix, or released locally by osteoblasts or mononuclear cells ${ }^{34}$ (fig 3). Figure 3 gives the proposed function of 1,25-dihydroxyvitamin $\mathrm{D}_{3}$, lymphokines, and cytokine receptors in bone remodelling and immunomodulation.

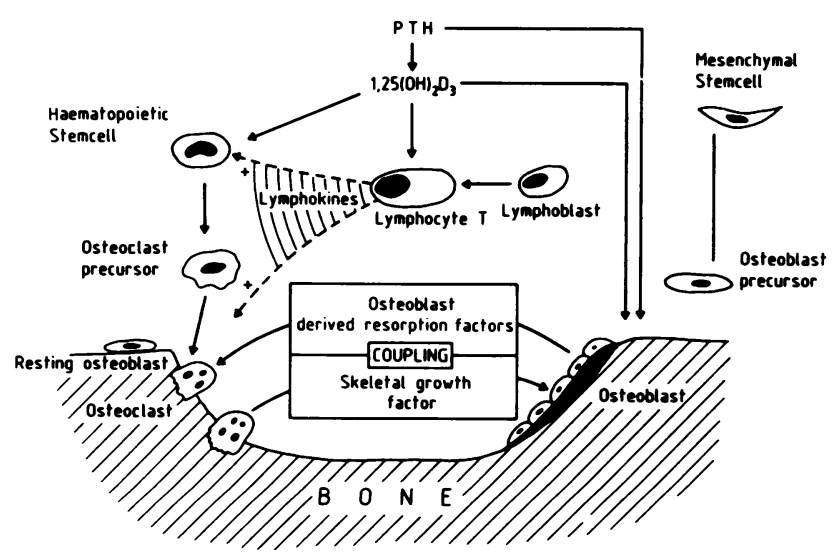

Figure 3: Proposed function of 1,25-dihydroxyvitamin $D_{3}\left(1,25(\mathrm{OH})_{2} \mathrm{D}_{3}\right.$, parathyroid hormone (PTH), lymphokines, and cytokines in bone remodelling and immunomodulation. Local growth factors: skeletal cell derived $S m C$ or IgF 1 , transforming growth factor, bone derived growth factor, or $\beta_{2}$ microglobulin, platelet derived growth factor, prostaglandin $E_{2}$, blood cell derived monokines (interleukin 1, tumour necrosis factor, macrophage derived growth factor, platelet derived growth factor, lymphokines (lymphotoxin or tumour necrosis factor, interferon), prostaglandins. Systemic factors: hormones $\left(P T H, 1,25(\mathrm{OH})_{2} D_{3}\right.$, calcitomin, corticosteroids, sex hormones, thyroxine, growth hormone).
Prostaglandins, monokines, interleukin 1, tumour necrosing factor with interferon gamma and lymphokines may be major regulators of bone destruction and repair in RA. Several systemic and local growth factors probably take part in the chronic inflammatory process, and stimulate bone destruction in addition to reparative processes. ${ }^{35}$

1,25-Dihydroxyvitamin $\mathrm{D}_{3}$ and retinoid acid affect monolymphokine regulation. Both factors are bone resorbing agents and they also regulate aspects of bone formation. Moreover, these agents enhance the release of interleukin 1 and regulate lymphokine release (interleukins 2 and 3), which may explain some of their actions on bone.

\section{New markers of bone mineral metabolism}

There is at present an intensive search for markers of bone mineral metabolism which will either help to explain the sequence of bone loss and bone remodelling or identify those persons at risk for osteoporosis. Useful information has been obtained by examination of the roles of collagenous and non-collagenous bone proteins in bone mineral metabolism. ${ }^{36}$

Urinary hydroxyproline is used as a marker of bone absorption because hydroxyproline once released from bone is not recycled for collagen biosynthesis. In addition, the proportion of hydroxyproline excreted in the bone seems to be constant so that the rate of excretion is a useful index of the rate of collagen degradation and bone turnover. Hydroxyproline excretion in RA is, however, also correlated with disease activity and not necessarily with bone loss. ${ }^{13}$

In osteoporosis urinary hydroxyproline is increased in 'fast bone losers' - that is, those patients in whom resorption is exaggerated, particularly at the menopause. It has recently been claimed that urinary hydroxyproline values, together with body fat index, alkaline phosphatase, and urinary calcium measurements, predict $79 \%$ of postmenopausal women with accelerated bone loss. ${ }^{37}$

Two hours' fasting morning calcium/creatinine ratio and hydroxyproline/creatinine ratio are the best markers at present for bone resorption because they are not influenced by diet.

\section{SERUM BONE $\gamma$-CARBOXYGLUTAMIC ACID PROTEIN- OSTEOCALCIN}

Bone $\gamma$-carboxyglutamic acid protein, also called osteocalcin, is the most abundant non-collagenous protein of bone matrix. Circulating bone $\gamma$-carboxyglutamic acid protein may be measured by specific radioimmunoassay. Bovine osteocalcin cross reacts with human osteocalcin. Several studies have shown that measurement of serum bone $\gamma$ carboxyglutamic acid protein provides a sensitive and useful marker of bone metabolism in a variety of metabolic bone diseases. At first it was thought that serum osteocalcin concentrations would reflect the amount of bone laid down because the protein is produced by osteoblasts. Because of the coupling between bone resorption (osteoclasts) and bone formation, however, raised osteocalcin concentrations are also found in conditions with a negative bone balance and a high turnover, as in osteoporosis and RA.

Serum osteocalcin shows diurnal variation-concentrations fall during the morning, rise in the afternoon and early evening, and reach a peak at night. ${ }^{38}$ Seasonal variation with the highest values during winter and the lowest during summer have been noted. Glucocorticosteroid treatment reduces serum osteocalcin concentrations and corticosteroid pulse therapy reduces these concentrations for a long time. ${ }^{39}$

Finally, local regulators of skeletal growth, which include polypeptide growth factors, bone and cartilage inducing factors, blood cell derived growth factors, and prostaglandins, 
may well assume greater importance in the pathogenesis of metabolic bone disease in the future. The actions of these factors, which have been studied mainly in vitro, have been recently reviewed. ${ }^{35}$

It seems that at present serum bone $\gamma$-carboxyglutamic acid protein is the one bone protein that promises to assist in the diagnosis and management of high turnover metabolic bone disease states. If further studies confirm its usefulness in osteoporosis as a predictor of rapid bone loss without the need for bone biopsy this serum marker will then not only allow early detection but also an appropriate choice of treatment in osteoporosis-that is, the use of specific inhibitors of high turnover states, such as oestrogen, calcitonin, or biphosphonates. In low turnover osteoporosis it may also be useful in determining whether the osteoblast may be stimulated to enhance bone formation with treatments such as fluoride, anabolic steroids, parathyroid hormone, 1,25-dihydroxyvitamin $\mathrm{D}$, etc. ${ }^{40}$

\section{Conclusions}

In patients with chronic arthritis there is no doubt that localised periarticular bone loss occurs owing to local disease activity, with release of local bone resorbing agents, and to loss of mobility. A general bone loss due to a systemic factor in RA is not substantiated with the present sophisticated non-invasive bone measurement techniques. Use of corticosteroids in the treatment of RA is an additional risk factor for loss of bone mass, especially in children and postmenopausal women. The possible influence of non-steroidal antiinflammatory drugs on the synthesis of local prostaglandin and of inhibitors of bone resorption, such as oestrogens, androgens, anabolic steroids, calcitonin, fluoride, and diphosphonates, on bone metabolism are promising new approaches to the reduction of bone lesions in chronic arthritis, but extensive further research is necessary. Development of precise tools to discover which patients are at risk of osteoporosis and to measure bone mass and bone metabolism changes over time will stimulate further research in this exciting multidisciplinary field.

\author{
Arthritis and Metabolic Bone Disease Research Unit \\ KU Leuven \\ UZ Pellenberg, \\ B-3041 Pellenberg, \\ Belgium
}

\section{J DEQUEKER}

P GEUSENS

1 Kaplan F S. Osteoporosis. West Caldwell, New Jersey: Ciba Geigy, 1983: (Ciba Clinical Symposium No 35).

2 Albright F, Reifenstein E. The parathyroid glands and metabolic bone disease. Selected studies. Baltimore: Williams and Wilkins, 1948: 393.

3 Dequeker J. The relationship between osteoporosis and osteoarthritis. Clin Rheum Dis 1985; 11: 271-96.

4 Dequeker J, Johnston C C, eds. Non-invasive bone measurements: methodological problems. Oxford, Washington DC: IRL Press, 1982: 256.

5 Dequeker J, Geusens $\mathrm{P}, \mathrm{W}$ ahner $\mathrm{H} W$, eds. Bone mineral measurements by photon absorptiometry: methodological problems. Leuven: Leuven University Press, 1988: 490.

6 Geusens P, Dequeker J. Photon absorptiometry: normal population data and fracture threshold. In: Ringe E F, Evans N D, Dixon A S, eds. Osteoporosis and bone mineral measurements. York: IPSM Publications, 1989: 91-7.

7 Barwell H. Disease of the joints. London: Hardwicke, 1865.

8 Soila P. Roentgen manifestations of adult rheumatoid arthritis. Acta Rheumatologica Scandinavica 1985: suppl 1.
9 McConkey B, Frazer G, Eligh A. Transparent skin and osteoporosis. A study in patients with rheumatoid disease. Ann Rheum Dis 1965; 24: 219-23.

10 Saville P D, Kharmosh P. Osteoporosis of rheumatoid arthritis: influence of age, sex and corticosteroids. Arthritis Rheum 1967;10: 423.

11 Kennedy A C, Lindsay R. Bone involvement in rheumatoid arthritis. Clin Rheum Dis 1977; 3: 403-20.

12 Bijlsma J W J. Bone metabolism in patients with rheumatoid arthritis. Clin Rheumatol 1988; 7: 16-23.

13 Mbuyi J M, Dequeker J, Teblick M, Merlevede M. Relevance of urinary excretion of alcian blue glycosaminoglycans complexes and hydroxyproline to disease activity in rheumatoid arthritis. $\mathcal{F}$ Rheumatol 1982; 9: 579-83.

14 Gevers G, Devos P, De Roo M, Dequeker J. Increased levels of osteocalcin (serum bone gla protein) in rheumatoid arthritis. $\mathrm{Br} \mathcal{F}$ Rheumatol 1986; 25: 260-2.

15 Verstraeten A, Dequeker J. Mineral metabolism in postmenopausal women with active rheumatoid arthritis. F Rheumatol 1986; 13: 43-6.

6 Rajapakse C, Thompson E, Grennan D H, et al. Increased bone metabolism in rheumatoid arthritis as measured by the whole body retention of ${ }^{4 \% \mathrm{~m}} \mathrm{Tc}$ methylene diphosphonate. Ann Rheum Dis 1983; 42: 138-41.

17 Verstraeten A, Dequeker J. Vertebral and peripheral bone mineral content and fracture incidence in postmenopausal patients with rheumatoid arthritis: effect of low dose corticosteroids. Ann Rheum Dis 1986; 45: 852-7.

18 Compston J E, Crawley E O, Evans C, O’Sullivan M M. Spinal trabecular bone mineral content in patients with non-steroid treated rheumatoid arthritis. Ann Rheum Dis 1988; 47: 660-4.

19 Mellish R W E, O'Sullivan M M, Garrahan N J, Compston J E. Iliac crest trabecular bone mass and structure in patients with non-steroid treated rheumatoid arthritis. Ann Rheum Dis 1987; 46: 830-6.

20 Mbuyi-Muamba J M, Dequeker J, Burssens A. Massive osteolysis in a case of rheumatoid arthritis: clinical, histologic and biochemical findings. Metabolic Bone Disease and Related Research 1983; 5: 101-6.

21 Nagant de Deuxchaisnes C, Devogelaer J P, Esselinckx W, et al. The effect of low dosage glucocorticoids on bone mass in rheumatoid arthritis: a crosssectional and longitudinal study using single photon absorptiometry. In: Avioli L, German C, Imbumbo $\mathrm{H}$, eds. Glucocorticoid effects and their biological consequences. New York: Plenum Press, 1984: 209-39.

22 Als O S, Gotfredsen A, Christiansen C. The effect of glucocorticosteroids on bone mass in rheumatoid arthritis. Arthritis Rheum 1985; 28: 369-75.

23 Nagant de Deuxchaisnes C, Devogelaer J P, Huaux J P. Influence of the menopausal state and the effect of low-dose glucocorticosteroids on bone mass in rheumatoid arthritis patients. Arthritis Rheum 1986; 29: 693-4.

24 Hajiroussou V J, Webley M. Prolonged low-dose corticosteroid therapy and osteoporosis in rheumatoid arthritis. Ann Rheum Dis 1984; 43: 24-7.

25 McConkey B, Fraser G M, Bligh A S. Osteoporosis and purpura in cheumatoid disease: prevalence and relation to treatment with corticosteroids. Qf Med 1962; 31: 419-27.

26 Zerwekh J E, Emkey R D, Harris E D. Low-dose prednisone therapy in rheumatoid arthritis: effect on vitamin D metabolism. Arthritis Rheum 1984; 27: $1050-2$.

27 Hahn T J, Halstead L E, Teitelbaum S L, Hahn B H. Altered mineral metabolism in glucocorticoid induced osteopenia: effect of 25-hydroxyvitamin D administration. $\mathcal{F}$ Clin Invest 1979; 64: 656-65.

28 Frumar A M, Meldrum D R, Geola F, et al. Relationship of fasting urinary calcium to circulating estrogen and body weight in postmenopausal women. f Clin Endocrinol Metab 1980; 50: 70-5.

29 Dykman $T R$, Haralson $K M$, Gluck $O S$, et al. Effect of oral 1,25dihydroxyvitamin $\mathrm{D}$ and calcium on glucocorticoid-induced osteopenia in patients with rheumatic diseases. Arthritis Rheum 1984; 27: 1336-43.

30 Baylink D J. Corticosteroid induced osteoporosis. N Engl f Med 1983; 309: 306-10.

31 Chesnut C H, Ivey J L, Gruber H E, et al. Stanozolol in postmenopausal osteoporosis: therapeutic efficacy and possible mechanisms of action. osteoporosis: therapeutic
Metabolism 1983; 32: 571 .

32 Geusens P, Dequeker J. Long-term effect of nandrolone decanoate, 1 alpha hydroxyvitamin D3 or intermittent calcium infusion therapy on bone mineral content, bone remodeling and fracture rate in symptomatic osteoporosis: a double-blind controlled study. Bone and Mineral 1986; 1 : $347-57$.

33 Kaplan A P. Kinins and bone resorption in rheumatic diseases. Arthritis Rheum 1987; 30: 589-92.

34 Haussler M R, Donaldson C A, Kelly M A, Mangelsdorf D J, Marion S L, Pike $J W$. Functions and mechanisms of action of the 1,25-dihydroxyvitamin D3 receptor. In: Norman A W, Schaefer K, Grigoleit H G, von Herrath D, eds. Vitamin D: a chemical, biochemical and clinical update. Berlin, New York: de Gruyter, 1985: 83-92.

35 Canalis E, McCarthy T, Centrella M. Growth factors and the regulation of bone remodeling. $\mathcal{f}$ Clin Invest 1988; 81: 277-81.

36 Mbuyi-Muamba J M, Dequeker J. Biochemistry of bone. In: Ballière's clinical rheumatology 1988; 2: 63-101.

37 Christiansen C, Riis B J, Rodbro P. Prediction of rapid bone loss in postmenopausal women. Lancet 1987; i: 1105

38 Gundberg C M, Markowitz M E, Mizruchi M, Rosen J F. Osteocalcin in human serum. A circadian rhythm. I Clin Endocrinol Metab 1985; 60: 736

39 Gevers G, Westhovens R, Dequeker J, Devos P, De Roo M. Effect of puls corticosteroid therapy on serum osteocalcin levels in rheumatoid arthritis. Clin Rheumatol 1987; 6: 125.

40 Epstein S. Serum and urinary markers of bone remodelling: assessment of bone turnover. Endocr Rev 1988; 9: 437-49. 